\title{
Collective Motion: Bistability and Trajectory Tracking
}

\author{
Derek Paley ${ }^{1}$ and Naomi Ehrich Leonard ${ }^{2}$ \\ Mechanical and Aerospace Engineering \\ Princeton University \\ Princeton, NJ 08544, USA \\ $\{$ dpaley $\{$ naomi $\}$ eprinceton.edu
}

\author{
Rodolphe Sepulchre ${ }^{3}$ \\ Electrical Engineering and Computer Science \\ Université de Liège \\ Institut Montefiore B28, B-4000 Liège, Belgium \\ r.sepulchredulg.ac.be
}

\begin{abstract}
This paper presents analysis and application of steering control laws for a network of self-propelled, planar particles. We explore together the two stably controlled group motions, parallel motion and circular motion, for modeling and design purposes. We show that a previously considered control law simultaneously stabilizes both parallel and circular group motion, leading to bistability and hysteresis. We also present behavior primitives that enable piecewise-linear network trajectory tracking.
\end{abstract}

\section{INTRODUCTION}

Collective motion is a compelling phenomenon whether observed in nature or in an engineering application. Two quantities that distinguish coordinated motion in a spatial network are the group linear and angular momentum. Parallel translation is characterized by large linear and small angular momentum about the center of mass, whereas rotation about a fixed center of mass has small linear and large angular momentum. Individual control laws that stabilize these two types of group motion are useful from both modeling and design perspectives.

Of particular interest for modeling purposes is a control law that stabilizes both parallel and circular group motions for the same control parameters, i.e. a bistable system. Bistability is responsible for a hysteresis behavior that has been observed in discrete simulations of fish schools as a function of a relative alignment coupling parameter [4]. These fish schooling results suggest that gyroscopic turning forces, i.e. those derived from vector potentials, may be better suited for a continuous model of this phenomenon than forces derived from scalar potentials. Another motivation for considering gyroscopic or steering controls is an analogy with coupled phase oscillators [11]. Powerful, analytical results exist for the synchronization of identical, sinusoidally coupled oscillators [12].

In addition to the modeling objectives, feedback laws that produce collective motion of particle groups have engineering applications such as unmanned sensor networks. For example, autonomous underwater vehicles (AUVs) are

\footnotetext{
${ }^{1}$ This work was supported by the Department of Defense National Defense Science and Engineering Graduate Fellowship, the Princeton University Gordon Wu Graduate Fellowship, and the Pew Program in Biocomplexity at Princeton University.

${ }^{2}$ Research partially supported by by NSF grant CCR-9980058, ONR grants N00014-02-1-0826 and N00014-02-1-0861, and AFOSR grant F49620-01-1-0382.

${ }^{3}$ This paper presents research results of the Belgian Programme on Interuniversity Attraction Poles, initiated by the Belgian Federal Science Policy Office. The scientific responsibility rests with its authors.
}

used to collect oceanographic measurements in formations that maximize the information intake, see e.g. [10]. A complementary approach to adaptive sampling is to identify a sensor coverage metric and design controls that maximize this objective, e.g. [3]. These approaches emphasize optimal trajectories; the coordination may be explicit as with artificial potentials or implicit as with strategies that minimize redundant coverage.

In this paper, we consider a kinematic model of identical, all-to-all coupled, planar particles. In a sensor network application, this represents an all-to-all communication topology. The particles move at constant speed and are subject to steering controls as described in Section II. We explore feedback controls that stabilize parallel and circular collective motion for modeling and design purposes. In Section III, we present stability results for a previously considered two particle steering control law, showing that it yields a bistable system: i.e. there is a parameter range for which the control law stabilizes both parallel and circular motion. In Section IV, we describe a procedure for piecewise-linear formation trajectory tracking using control laws that stabilize parallel or circular motion as building blocks. We plan to extend these results in the future along both the modeling and design themes.

\section{PARTICLE MODEL}

We study a continuous-time, kinematic model of $N$ identical, self-propelled particles subject to planar steering controls, following [6], [7]. In complex notation, the model is given by

$$
\begin{aligned}
& \dot{\mathbf{r}}_{k}=e^{i \theta_{k}} \\
& \dot{\theta}_{k}=u_{k},
\end{aligned}
$$

where $\mathbf{r}_{k} \in \mathbb{R}^{2}$ and $\theta_{k} \in S^{1}$ are the position and heading of the $k$ th particle. Unless otherwise indicated, $k=1, \ldots, N$. The steering control law is denoted by $u_{k}$. If we define the relative position and orientation variables, $\mathbf{r}_{j k}=\mathbf{r}_{j}-\mathbf{r}_{k}$ and $\theta_{j k}=\theta_{j}-\theta_{k}$, then the control, $u_{k}$, can be decomposed into relative spacing and alignment terms, i.e.

$$
u_{k}=u_{k}^{s p a c}\left(\mathbf{r}_{j k}, \theta_{j k}\right)+u_{k}^{\text {align }}\left(\theta_{j k}\right) .
$$

The alignment control is a function of the relative orientation, $\theta_{j k}$, whereas the spacing control is function of both the relative position, $\mathbf{r}_{j k}$, and orientation, $\theta_{j k}$. 
Throughout the paper, we consider relative alignment control of the form,

$$
u_{k}^{\text {align }}=\frac{K}{N} \sum_{j=1}^{N} \sin \theta_{j k},
$$

where $K \in \mathbb{R}$ is a control gain. The gain $K$ plays an important role in the paper: we present new analysis for $|K|=O(1)$ in Section III and apply previous results for $|K| \gg 0$ in Section IV.

Global convergence results for the model (1)-(2) are presented in [11]. In this earlier work, we provide feedback controls to stabilize parallel and circular collective motion by considering the singularly perturbed system,

$$
\begin{aligned}
\dot{\mathbf{r}}_{k} & =e^{i \theta_{k}} \\
\varepsilon \dot{\theta}_{k} & =\varepsilon u_{k}^{s p a c}\left(\mathbf{r}_{j k}, \theta_{j k}\right)+\frac{1}{N} \sum_{j=1}^{N} \sin \theta_{j k},
\end{aligned}
$$

where $\varepsilon=\frac{1}{K}$ is a small parameter. This approach decouples the spacing and alignment control terms into slow and fast time scales, respectively. The fast subsystem has the form of a network of coupled phase oscillators, i.e.

$$
\dot{\theta}_{k}=\omega_{k}+\frac{\operatorname{sgn}(K)}{N} \sum_{j=1}^{N} \sin \theta_{j k},
$$

where all oscillators have the same natural frequency, $\omega_{k}=$ $\omega_{o}$. In a coordinate frame rotating at $\omega_{o}, \omega_{k}=0$.

Watanabe and Strogatz, [12], utilize a coordinate transformation to deduce $N-3$ constants of motion for the model (7). Consequently, they prove that the oscillators synchronize for $K>0$ and converge (anti-synchronize) to an incoherent state for $K<0$. In the particle model, the parameter $K$ drives the group linear momentum to its maximum for $K>0$ and to zero for $K<0$ [11]. This corresponds to parallel motion and rotation about a fixed center of mass, respectively. In the slow time scale, spacing controls refine these two types of trajectories. We provide spacing controls that support the design of network trajectory tracking in Section IV.

\section{BISTABLE CONTROL LAW}

In this section, we study a two particle system, i.e. $N=2$, of the form (1)-(4) with $|K|=O(1)$. We consider a rectilinear control law that was presented with convergence results in [6]. Using complex notation, the spacing controls are given by

$$
\begin{aligned}
u_{1}^{s p a c} & =-<\frac{\mathbf{r}_{21}}{\rho}, i e^{i \theta_{1}}>\left(<\frac{\mathbf{r}_{21}}{\rho}, e^{i \theta_{1}}>+f(\rho)\right) \\
u_{2}^{s p a c} & =-<\frac{\mathbf{r}_{12}}{\rho}, i e^{i \theta_{2}}>\left(<\frac{\mathbf{r}_{12}}{\rho}, e^{i \theta_{2}}>+f(\rho)\right),
\end{aligned}
$$

where $\left\langle\cdot, \cdot>\right.$ is the scalar product and $\rho=\left\|\mathbf{r}_{12}\right\|=\left\|\mathbf{r}_{21}\right\|$. The function $f(\rho)$ is defined as in [6],

$$
f(\rho)=1-\left(\frac{\rho_{o}}{\rho}\right)^{2},
$$

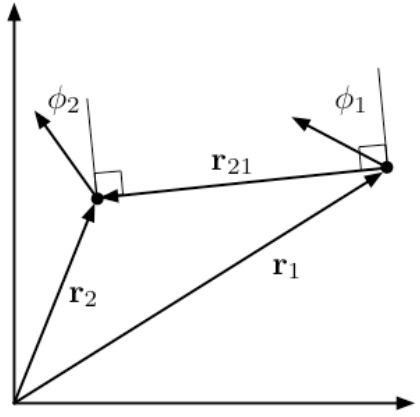

Fig. 1. Shape coordinates for a two particle system, where $\rho=\left\|\mathbf{r}_{21}\right\|$, from [6].

where $\rho_{o}>0$ is a constant spacing parameter. The first terms in the parentheses in (8)-(9) are zero for either clockwise or counter-clockwise rotation of the particle pair about its center of mass. The second terms are zero for the relative spacing $\rho=\rho_{o}$.

Using the shape coordinates illustrated in Figure 1, the system with the spacing controls (8)-(9) becomes

$$
\begin{aligned}
\dot{\rho}= & \sin \phi_{2}-\sin \phi_{1} \\
\dot{\phi}_{1}= & -\sin \phi_{1} \cos \phi_{1}+f(\rho) \cos \phi_{1} \\
& +\frac{K}{2} \sin \left(\phi_{2}-\phi_{1}\right)+\frac{1}{\rho}\left(\cos \phi_{2}-\cos \phi_{1}\right) \\
\dot{\phi}_{2}= & -\sin \phi_{2} \cos \phi_{2}-f(\rho) \cos \phi_{2} \\
& +\frac{K}{2} \sin \left(\phi_{1}-\phi_{2}\right)+\frac{1}{\rho}\left(\cos \phi_{2}-\cos \phi_{1}\right),
\end{aligned}
$$

where $\phi_{1}, \phi_{2} \in S^{1}$ [6]. This control law is specified entirely in terms of the relative distance and orientation coordinates, $\rho, \phi_{1}$, and $\phi_{2}$. As shown in [6], there are only two types of relative equilibria of the system: parallel and circular motion. The parallel motion can be divided into two categories: translation perpendicular to the baseline vector, $\mathbf{r}_{12}$, and leader-follower motion with arbitrary separation.

The fixed points for the system (11)-(13) that correspond to these relative equilibria are given as follows: $\left(\rho_{o}, 0,0\right)$ and $\left(\rho_{o}, \pi, \pi\right)$ produce parallel translation perpendicular to the baseline vector, $\mathbf{r}_{12}$, with $\left\|\mathbf{r}_{12}\right\|=\rho_{o} ;\left(\rho_{2}, \pi, 0\right)$ and $\left(\rho_{2}, 0, \pi\right)$ produce clockwise and counter-clockwise rotation about the center of mass at radius, $\rho_{2}$, determined by the roots of $f\left(\rho_{2}\right)-\frac{2}{\rho_{2}}=0$; and $\left(\rho, \frac{\pi}{2}, \frac{\pi}{2}\right)$ and $\left(\rho, \frac{3 \pi}{2}, \frac{3 \pi}{2}\right)$ produce a leader-follower formation at an arbitrary separation distance, $\rho$. In the next section, we determine the local stability of these relative equilibria using linearization about each fixed point.

\section{A. Linear Stability Analysis}

We derive the stability properties of the three relative equilibria of the two particle control law (8)-(9) as a function of the control gain, $K$, by linearizing (11)-(13) at the corresponding fixed points. Starting with the parallel relative equilibrium, the characteristic polynomial of the 
Jacobian evaluated at either $\left(\rho_{o}, 0,0\right)$ or $\left(\rho_{o}, \pi, \pi\right)$ is

$$
\left(\lambda_{1}+1\right)\left(\lambda^{2}+(1+K) \lambda+\frac{4}{\rho_{o}}\right)=0,
$$

where the roots of the quadratic term are $\lambda_{2}$ and $\lambda_{3}$. Thus we have $\lambda_{1}=-1$. In addition, for $K>-1, \Re\left(\lambda_{2,3}\right)<0$; at $K=-1, \mathfrak{R}\left(\lambda_{2,3}\right)=0$; and for $K<-1, \mathfrak{R}\left(\lambda_{2,3}\right)>0$.

Similarly, the characteristic polynomial for both circular relative equilibria, i.e. $\left(\rho_{2}, \pi, 0\right)$ and $\left(\rho_{2}, 0, \pi\right)$, is given by

$$
\left(\lambda_{1}+1-K\right)\left(\lambda^{2}+\lambda+4 g\left(\rho_{o}\right)\right)=0,
$$

where

$$
g\left(\rho_{o}\right)=\frac{\rho_{o}^{2}}{\rho_{2}^{2}}+\frac{1}{\rho_{2}^{2}}>0,
$$

and the roots of the quadratic term in (15) are $\lambda_{2}$ and $\lambda_{3}$. Thus we have $\Re\left(\lambda_{2,3}\right)<0$. In addition, for $K<1, \lambda_{1}<0$; at $K=1, \lambda_{1}=0$; and for $K>1, \lambda_{1}>0$.

Lastly, the characteristic polynomial for the leaderfollower relative equilibria, i.e. $\left(\rho, \frac{\pi}{2}, \frac{\pi}{2}\right)$ or $\left(\rho, \frac{3 \pi}{2}, \frac{3 \pi}{2}\right)$, is given by

$$
\lambda_{1}\left(\lambda^{2}+(K-2) \lambda+\frac{1}{2}(h(\rho)-K)\right)=0,
$$

where

$$
h(\rho)=1-f^{2}(\rho)+\frac{2}{\rho} f(\rho),
$$

and the roots of the quadratic term in (17) are $\lambda_{2}$ and $\lambda_{3}$. Thus we have $\lambda_{1}=0$. In addition, for $K<2, \Re\left(\lambda_{2,3}\right)>0$.

Proposition 1: The two particle control law, (8)-(9), is a bistable system for $|K|<1$ and the two stable equilibria are parallel and circular motion.

Proof: Parallel motion is asymptotically stable for $K>$ -1 because the Jacobian is a Hurwitz matrix. It is unstable for $K<-1$ because $\mathfrak{R}\left(\lambda_{2,3}\right)>0$. Similarly, circular motion is asymptotically stable for $K<1$ because the Jacobian is a Hurwitz matrix. It is unstable for $K>1$ because $\lambda_{1}>0$. Finally, the leader-follower motion is unstable for $K<2$ because $\Re\left(\lambda_{2,3}\right)>0$.

Remark 1: We can classify the bifurcations that occur at $K= \pm 1$. For the parallel relative equilibria, we observe that a pair of complex eigenvalues, $\lambda_{2}$ and $\lambda_{3}$, passes through the imaginary axis as $K$ decreases through $K=-1$. We conclude from the results of the Lyapunov analysis below that this is a subcritical Hopf bifurcation. For the circular relative equilibria, we observe that $\lambda_{1} \in \mathbb{R}$ passes through zero as $K$ increases through $K=1$ which yields a saddlenode bifurcation.

\section{B. Lyapunov Analysis}

For the control law (8)-(9), we show almost global convergence to the set of parallel relative equilibria with $K>1$ and almost global convergence to the set of circular relative equilibria with $K<-1$. Using the results of [6], we consider the Lyapunov function,

$$
V=-\log \left(\cos \left(\phi_{2}-\phi_{1}\right)+1\right)+F(\rho)
$$

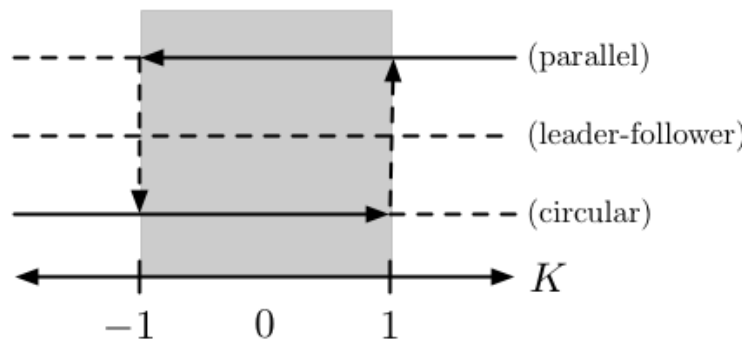

Fig. 2. Hysteresis characteristics of the two particle control law for $|K|=O(1)$. The horizontal solid (dashed) lines indicate stable (unstable) relative equilibria. The grey box indicates the bistable region.

where $f(\rho)=\frac{d F}{d \rho}$ and $\left|\phi_{2}-\phi_{1}\right| \neq \pi$. The time derivative of $V$ along the solutions of (11)-(13) is

$$
\dot{V}=-\frac{\sin ^{2}\left(\phi_{1}-\phi_{2}\right)}{\cos \left(\phi_{2}-\phi_{1}\right)+1}\left(K+\cos \left(\phi_{1}+\phi_{2}\right)\right),
$$

which is non-positive for $K>1$ and non-negative for $K<$ -1 . For $K>1$, the Lasalle invariance principle can be used to prove that all solutions converge either to the set of parallel relative equilibria, where $V$ is minimum, or to the set of (unstable) circular or leader-follower equilibria. Likewise, for $K<-1$, the function $W=e^{-V}$ is nonincreasing along the solutions, and the Lasalle invariance principle can be used to prove that all bounded solutions converge either to the set of circular relative equilibria, where $W$ is minimum, or to the set of (unstable) parallel or leaderfollower equilibria.

Remark 2: The almost global convergence to parallel equilibria for $K>1$ and to circular equilibria for $K<-1$, combined with the bistability of the parallel and circular equilibria in the parameter range $K \in[-1,1]$ causes a hysteresis behavior under slow variation of $K=0(1)$ [8]. The hysteresis characteristics are illustrated in Figure 2.

\section{TRAJECTORY TRACKING}

In this section we apply previous results for the design of feedback control laws for parallel and circular collective motion [11]. In particular, we use feedback controls in conjunction with impulsive controls to achieve trajectory tracking of the center of mass of the network. The admissible reference inputs are piecewise-linear paths. Note that we use different controls for parallel vs. circular control as opposed to the single control law of Section III.

\section{A. Collective Motion Feedback Controls}

The collective motion feedback controls are based on the singularly perturbed model, (5)-(6). In [11], we provide feedback control laws to stabilize parallel and circular collective motion. The feedback control laws are separated into relative spacing and alignment control terms, as in (3). Both parallel and circular controllers use the alignment control given in (4), with $K \gg 0$ and $K \ll 0$, respectively. In this section, we provide spacing controls that are applicable to trajectory tracking. 
1) Parallel Motion: For $K \gg 0$, all particle headings asymptotically synchronize (to first order) in the fast time scale [11]. The design of a controller that stabilizes approximate uniform relative spacing is given in [11] and is summarized below.

The slow manifold of (6) is given by

$$
\theta_{k}=\theta_{1}+\varepsilon\left(u_{k}^{s p a c}-u_{1}^{s p a c}\right)+O\left(\varepsilon^{2}\right),
$$

where $\varepsilon=\frac{1}{K}>0$. Substituting (21) into (5) gives the slow dynamics

$$
\dot{\mathbf{r}}_{k}=e^{i \theta_{1}}\left(1+\varepsilon i\left(u_{k}^{s p a c}-u_{1}^{s p a c}\right)\right)+O\left(\varepsilon^{2}\right) .
$$

Solving for $\dot{\mathbf{r}}_{k j}=\dot{\mathbf{r}}_{k}-\dot{\mathbf{r}}_{j}$, we obtain the slow dynamics for the particle spacing, which are given by

$$
\dot{\mathbf{r}}_{k j}=\varepsilon i e^{i \theta_{1}}\left(u_{k}^{s p a c}-u_{j}^{s p a c}\right)+O\left(\varepsilon^{2}\right) .
$$

To design the formation spacing control we follow the approach developed in [1]. Consider the following interparticle scalar potential,

$$
U_{I}\left(\mathbf{r}_{k j}\right)=\log \left\|\mathbf{r}_{k j}\right\|+\frac{\rho_{o}}{\left\|\mathbf{r}_{k j}\right\|},
$$

which is an even function of $\mathbf{r}_{k j}$. Using $\frac{\partial \mathbf{r}_{k j}}{\partial \mathbf{r}_{k}}=1$, the gradient of this potential is given by

$$
\nabla U_{I}\left(\mathbf{r}_{k j}\right)=\left(\frac{1}{\left\|\mathbf{r}_{k j}\right\|}-\frac{\rho_{o}}{\left\|\mathbf{r}_{k j}\right\|^{2}}\right) \frac{\mathbf{r}_{k j}}{\left\|\mathbf{r}_{k j}\right\|} .
$$

We define the formation spacing control in terms of the negative of this gradient, i.e.

$$
u_{k}^{s p a c}=-\sum_{j \neq k}^{N}<\nabla U_{I}\left(\mathbf{r}_{k j}\right), i e^{i \theta_{k}}>.
$$

Consider the following Lyapunov function candidate,

$$
U=\sum_{k=1}^{N} \sum_{j>k}^{N} U_{I}\left(\mathbf{r}_{k j}\right) .
$$

Using (21)-(26), the time-derivative of $U$ (limited to first order terms in $\varepsilon$ ) is,

$$
\dot{U}=-\varepsilon \sum_{k=1}^{N}\left(u_{k}^{s p a c}\right)^{2},
$$

where we used the property that $\nabla U_{I}\left(\mathbf{r}_{k j}\right)$ is an odd function of $\mathbf{r}_{k j}$.

Proposition 2: The steering control law of the form (3), with $u_{k}^{\text {spac }}$ given by (26) and $u_{k}^{\text {align }}$ given by (4) with $K \gg 0$, stabilizes parallel motion with spacings that minimize the potential (27).

Proof: Parallel motion with approximate uniform spacing corresponds to the set of relative equilibria of (5)-(6) and (26), characterized by $\Gamma=\left\{\left(\mathbf{r}_{k}, \theta_{k}\right) \mid \theta_{k}=\right.$ $\left.\theta_{1}, U\left(\mathbf{r}_{k j}\right) \min , k=1, \ldots, N\right\}$. Exponential stability of $\Gamma$ for the singularly perturbed system follows from exponential stability of the set of synchronized states $\theta_{k}=\theta_{1}$ for the fast dynamics (7) with $K>0$ and exponential stability of the set

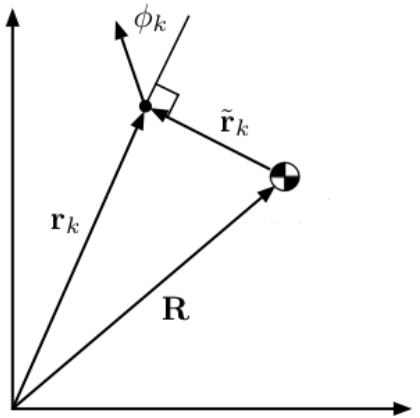

Fig. 3. Shape coordinates used for the circular feedback control, where $\rho_{k}=\left\|\tilde{\mathbf{r}}_{k}\right\|$ and $\mathbf{R}$ is the center of mass of the group, after [6].

of formations that minimize $U$ for the slow dynamics (23). Linearization at any of these equilibria shows neutral modes in the directions tangent to the set of equilibria (symmetry directions) and stable modes in the transverse directions.

2) Circular Motion: Setting $K \ll 0$ in (4) drives the particle phases in the fast time scale to the incoherent state, or balanced manifold, which corresponds to zero linear momentum [11]. Therefore, in the slow time scale, the spacing controls may utilize the center of mass as a fixed beacon. The spacing controls are subject to the constraint of preserving zero total linear momentum. As described in [11], spacing controls which preserve exact invariance of the balanced manifold, $u_{k}^{s p a c}$, are generated by projection of the so-called balanced controls, $u_{k}^{b a l}$. Simulations show that even in the absence of projection, the balanced controls achieve near-invariance of the balanced manifold, which is sufficient for the trajectory tracking objective.

The particles move in a circular trajectory about the center of mass, $\mathbf{R}=\frac{1}{N} \sum_{j=1}^{N} \mathbf{r}_{j}$. We define the vector from the center of mass to particle $k$ by $\tilde{\mathbf{r}}_{k}=\mathbf{r}_{k}-\mathbf{R}$, and its magnitude by $\rho_{k}=\left\|\tilde{\mathbf{r}}_{k}\right\|$, as shown in Figure 3. Consider the following balanced control, which is a variant of a single particle/beacon control law from [6],

$$
u_{k}^{b a l}=-f\left(\rho_{k}\right)<\frac{\tilde{\mathbf{r}}_{k}}{\rho_{k}}, i e^{i \theta_{k}}>-<\frac{\tilde{\mathbf{r}}_{k}}{\rho_{k}}, e^{i \theta_{k}}>,
$$

where $f\left(\rho_{k}\right)$ is given by

$$
f\left(\rho_{k}\right)=1-\left(\frac{\rho_{o}}{\rho_{k}}\right)^{2} .
$$

In shape coordinates, the dynamics for particle $k$ are

$$
\begin{aligned}
& \dot{\rho}_{k}=\sin \phi_{k} \\
& \dot{\phi}_{k}=-\left(f\left(\rho_{k}\right)-\frac{1}{\rho_{k}}\right) \cos \phi_{k}-\sin \phi_{k} \cos \phi_{k} .
\end{aligned}
$$

Convergence results for a system similar to (31)-(32) are presented in [6]. A Lyapunov function candidate for the system of $N$ particles is the sum of individual functions, $V_{I}$, given by

$$
V_{I}\left(\rho_{k}, \phi_{k}\right)=-\log \left(\left|\cos \phi_{k}\right|\right)+H\left(\rho_{k}\right),
$$


where $f\left(\rho_{k}\right)-\frac{1}{\rho_{k}}=\frac{d H}{d \rho_{k}}$. The time derivative of $V_{I}$ along the trajectories of (31) and (32) is given by

$$
\begin{aligned}
\dot{V}_{I} & =\left(\frac{\sin \phi_{k}}{\cos \phi_{k}}\right) \dot{\phi}_{k}+\left(f\left(\rho_{k}\right)-\frac{1}{\rho_{k}}\right) \dot{\rho}_{k} \\
& =-\frac{\sin ^{2} \phi_{k}}{\cos \phi_{k}} .
\end{aligned}
$$

Therefore, $\dot{V}_{I} \leq 0$ in the set $E=\left\{\left(\rho_{k}, \phi_{k}\right)\left|\rho_{k}>0,\right| \phi_{k} \mid<\frac{\pi}{2}\right\}$. The largest invariant set in $E$ for which $\dot{V}_{I}=0$ is $\left(\rho_{k}, \phi_{k}\right)=$ $\left(\rho_{1}, 0\right)$, where

$$
f\left(\rho_{1}\right)-\frac{1}{\rho_{1}}=0 .
$$

and $f\left(\rho_{k}\right)$ is given by (30) [6].

Proposition 3: The steering control law of the form (3), with $u_{k}^{\text {spac }}$ determined by (29) with the projection and $u_{k}^{\text {align }}$ given by (4) with $K \ll 0$ stabilizes clockwise circular motion about a fixed center of mass with radius, $\rho_{1}$, determined by (35).

Proof: Circular motion about a fixed center of mass corresponds to the set of relative equilibria of (5)-(6) and (29), characterized by $\Gamma=\left\{\left(\mathbf{r}_{k}, \theta_{k}\right) \mid \dot{\mathbf{R}}=0,\left\|\tilde{\mathbf{r}}_{k}\right\|=\rho_{1}, k=\right.$ $1, \ldots, N\}$. Exponential stability of $\Gamma$ for the singularly perturbed system follows from exponential stability of the balanced manifold, $\dot{\mathbf{R}}=0$, for the fast dynamics (7) with $K<0$ and exponential stability of the set of ring formations $\left\|\tilde{\mathbf{r}}_{k}\right\|=\rho_{1}$ for the (decoupled) slow dynamics (31)-(32). Exponential stability of the balanced manifold follows from the Lyapunov analysis in [12]. Exponential stability of the set of ring formations follows from exponential stability of the rotation equilibrium about the fixed center of mass for each particle, a consequence of the Lyapunov analysis in (33) and (34). Convergence to the balanced manifold is almost global for the fast dynamics: only synchronized solutions do not converge to the balanced manifold [12]. Convergence to the set of ring formations is almost global for the slow dynamics from the Lyapunov analysis. For the singularly perturbed system, the convergence results are therefore semiglobal, i.e. the conclusions for the fast and slow dynamics are asymptotically recovered as the small parameter $\varepsilon$ decreases to zero.

Remark 3: The second term in (29) stabilizes motion perpendicular to $\tilde{\mathbf{r}}_{k}$. Changing the sign of this term stabilizes counter-clockwise rotation, i.e.

$$
u_{k}^{b a l}=-f\left(\rho_{k}\right)<\frac{\tilde{\mathbf{r}}_{k}}{\rho_{k}}, i e^{i \theta_{k}}>+<\frac{\tilde{\mathbf{r}}_{k}}{\rho_{k}}, e^{i \theta_{k}}>.
$$

In shape coordinates, this control law is given by

$$
\dot{\phi}_{k}=-\left(f\left(\rho_{k}\right)-\frac{1}{\rho_{k}}\right) \cos \phi_{k}+\sin \phi_{k} \cos \phi_{k} .
$$

To see that this stabilizes counter-clockwise motion, observe that the time derivative of (33) becomes

$$
\dot{V}_{I}=\frac{\sin ^{2} \phi_{k}}{\cos \phi_{k}} .
$$

Therefore, $\dot{V}_{I} \leq 0$ in the set $E=\left\{\left(\rho_{k}, \phi_{k}\right)\left|\rho_{k}>0,\right| \phi_{k} \mid>\frac{\pi}{2}\right\}$. The largest invariant set in $E$ for which $\dot{V}_{I}=0$ is $\left(\rho_{k}, \phi_{k}\right)=$ $\left(\rho_{1}, \pi\right)$, where $\rho_{1}$ is determined by $(35)$.

\section{B. Behavior Primitives}

The parallel and circular feedback controls are used to define five behavior primitives which can be combined to track piecewise-linear trajectories. The behavior primitives use impulsive controls to align the particles with the reference input and the feedback controls to stabilize this trajectory. The behaviors will be referred to as random-tocircular, circular-to-parallel, parallel-to-parallel, parallelto-circular, and circular-to-circular. In parallel motion, the network center of mass follows a linear reference trajectory. In the circular state, the network center of mass is fixed, i.e. the group is stopped.

1) Random-to-circular: Starting from random initial conditions, this behavior stabilizes circular motion about a fixed center of mass. The input to this behavior is the spacing parameter, $\rho_{o}$, which determines the formation radius according to (35). The impulse control used to align the particles in the rotation direction is given by

$$
\triangle \theta_{k}=\arg \left( \pm i \tilde{\mathbf{r}}_{k}\right)-\theta_{k},
$$

where the \pm corresponds to counter- and clockwise rotation, respectively. The feedback control used to stabilize circular motion is of the form (3), where $u_{k}^{s p a c}$ is given by (29) for clockwise or (36) for counter-clockwise rotation. Note that we use the balanced spacing control, $u_{k}^{s p a c}=u_{k}^{b a l}$, i.e. we neglect the projection. The alignment control term, $u_{k}^{\text {align }}$, is given by (4) with $K \ll 0$.

2) Circular-to-parallel: Starting from circular motion, this behavior stabilizes parallel motion along a reference trajectory with approximate uniform particle spacing. The input to this behavior is the spacing parameter, $\rho_{o}$, which determines the critical points of the interparticle potential (24), and the reference heading, $\theta_{o}$. The impulse control which aligns the particles in the reference direction is

$$
\triangle \theta_{k}=\theta_{o}-\theta_{k}
$$

The feedback control that stabilizes parallel motion is of the form (3), where $u_{k}^{\text {spac }}$ is given by (26) and $u_{k}^{\text {align }}$ by (4) with $K \gg 0$.

3) Parallel-to-parallel: Starting from parallel motion, this behavior stabilizes parallel motion along a different reference trajectory. The input to this behavior is the new reference heading, $\theta_{o}$. The impulsive control used to align the particles in the input direction is given by (40). The feedback control used to stabilize parallel motion is of the form (3), where $u_{k}^{\text {spac }}$ is given by (26) and $u_{k}^{\text {align }}$ by (4) with $K \gg 0$.

4) Parallel-to-circular: Starting from parallel motion, this behavior stabilizes circular motion about a fixed center of mass. The input to this behavior is the spacing parameter, $\rho_{o}$, which determines the formation radius according to (35). The impulsive control used to align the particles in the 


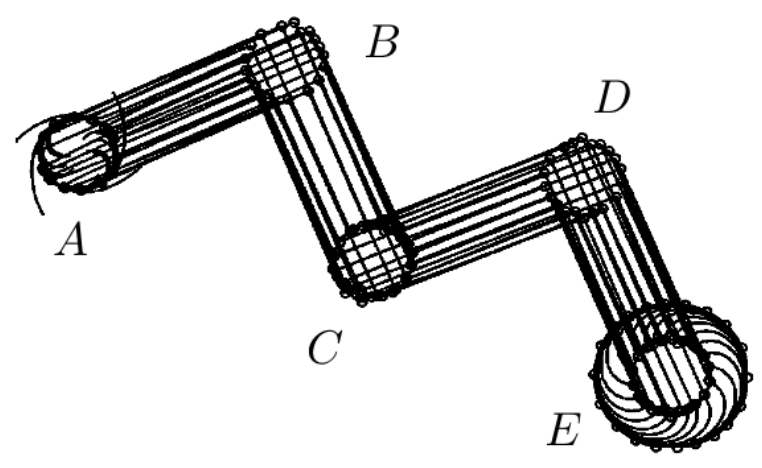

Fig. 4. Trajectory tracking with $N=20$ starting from random initial conditions. The reference input is a piecewise-linear curve. The behavior sequence starts in the vicinity of A with random-to-circular and then follows A circular-to-parallel, B parallel-to-parallel, and C parallel-tocircular. This sequence repeats for the points $\mathrm{C}, \mathrm{D}$, and $\mathrm{E}$ and then ends with the circular-to-circular behavior at $\mathrm{E}$.

rotation direction is given by (39). The feedback control used to stabilize circular motion is of the form (3), where $u_{k}^{\text {spac }}=u_{k}^{\text {bal }}$ is given by (29) or (36) and $u_{k}^{\text {align }}$ by (4) with $K \ll 0$.

5) Circular-to-circular: Starting from circular motion, this behavior stabilizes circular motion with a different radius, i.e. dilation/contraction. The input to this behavior is the new spacing parameter, $\rho_{o}$, which determines the formation radius according to (35). There is no impulsive control used to realign the particles. The feedback control used to stabilize circular motion is of the form (3), where $u_{k}^{s p a c}=u_{k}^{b a l}$ is given by (29) or (36) and $u_{k}^{\text {align }}$ by (4) with $K \ll 0$.

\section{Trajectory Tracking Example}

In this section, we use the behavior primitives to construct a behavior sequence that tracks an input reference trajectory. The admissible references are piecewise-linear paths specified by a list of desired heading and duration pairs. An example of trajectory tracking is shown in Figure 4.

In this example, twenty particles start from random initial conditions in the vicinity of the point $\mathrm{A}$. We observe that in simulation, $|K|=O(1)$ is sufficient to stabilize the desired collective motion. The behavior sequence starts with random-to-circular with $K=-1$ and $\rho_{o}=25$. This stabilizes circular motion at radius $\rho_{1}=25.5$ determined by (35). The next behavior in the sequence is circular-toparallel with parameters $K=1, \rho_{o}=25.5$, and $\theta_{o}=22.5^{\circ}$, which takes the sensor network from point $\mathrm{A}$ to point $\mathrm{B}$ in Figure 4. At point $\mathrm{B}$, the behavior parallel-to-parallel with $K=1$ is used to track the reference input $\theta_{o}=-67.5^{\circ}$ to point $\mathrm{C}$. Then the parallel-to-circular behavior stabilizes circular motion about a fixed center of mass with $K=-1$ and $\rho_{o}=25$. The sequence is repeated for the points $\mathrm{C}, \mathrm{D}$, and E. Lastly, the circular-to-circular behavior stabilizes circular motion with the radius $\rho_{o}=50\left(\rho_{1}=50.5\right)$ using $K=-1$.

\section{CONCLUSIONS AND FUTURE WORK}

In this paper, we extend the analysis of a previously considered two particle control law to show that it is bistable for $|K|=O(1)$, where $K$ is the relative alignment coupling parameter. This behavior is preserved in numerical simulations for many particles. However, for $N>2$, the control law stabilizes both clockwise and counter-clockwise rotations, which is undesirable for modeling natural groups, e.g. fish schools. We plan to complete the analysis for the two particle system and investigate the stability properties of a many particle system governed by a modified control scheme.

We also apply previous results for feedback control of networks of many particles to trajectory tracking of piecewise-linear paths. We use impulsive controls to input the reference trajectory and feedback controls to stabilize both parallel motion along the reference heading and circular motion around a fixed center of mass. We plan to extend the circular control law so that it is possible to stabilize particular configurations (formations) within the family of incoherent states, see e.g. [2]. For example, in the splay state, the particles have uniform phase differences, which corresponds to regular spacing around the circle. The dilation/contraction behavior may be extended to perform more elaborate formation shape control. We also plan to consider robustness to perturbations in the network topology such as those explored in [5] and [9].

\section{REFERENCES}

[1] R. Bachmayer and N. E. Leonard. Vehicle networks for gradient descent in a sampled environment. In Proc. IEEE Conf. on Decision and Control, 2002.

[2] E. Brown, P. Holmes, and J. Moehlis. Globally coupled oscillator networks. In Perspectives and Problems in Nonlinear Science: A Celebratory Volume in Honor of Larry Sirovich. Springer, 2003.

[3] J. Cortès, S. Martìnez, T. Karatas, and F. Bullo. Coverage control for mobile sensing networks. IEEE Trans. on Robotics and Automation, 20(2), 2004.

[4] I. D. Couzin, J. Krause, R. James, G. D. Ruxton, and N. R. Franks. Collective memory and spatial sorting in animal groups. J. theor. Biol., 218:1-11, 2002.

[5] A. Jadbabaie, J. Lin, and A. S. Morse. Coordination of groups of mobile autonomous agents using nearest neighbor rules. IEEE Trans. on Automatic Control, 48(6):988-1001, 2003.

[6] E. W. Justh and P. S. Krishnaprasad. A simple control law for UAV formation flying. Technical Report 2002-38, Institute for Systems Research, University of Maryland, 2002.

[7] E.W. Justh and P.S. Krishnaprasad. Steering laws and continuum models for planar formations. In Proc. IEEE Conf. Decision and Control, 2003.

[8] H. K. Khalil. Nonlinear Systems. Prentice Hall, third edition, 2002.

[9] J.A. Marshall, M.E. Broucke, and B.A. Francis. Unicycles in cyclic pursuit. In Proc. 2004 American Control Conf., pages 5344-5349, 2004.

[10] P. Ögren, E. Fiorelli, and N. E. Leonard. Cooperative control of mobile sensor networks: Adaptive gradient climbing in a distributed environment. IEEE Trans. on Automatic Control, 49(8):1292-1302, 2004

[11] R. Sepulchre, D. Paley, and N. Leonard. Collective motion and oscillator synchronization. In V.J. Kumar, N.E. Leonard, and A.S. Morse, editors, Proc. Block Island Workshop on Cooperative Control, June 2003.

[12] S. Watanabe and S. Strogatz. Constants of motion for superconductor arrays. Physica D, 74:197-253, 1994. 